\title{
Assessment of the effectiveness of standardized infusion devices for healthcare management
}

\author{
Shio Sugita ${ }^{1^{*}}$, Hideko Aida $^{2}$, Aya Okada $^{3}$, Hiroyuki Kobayashi ${ }^{2}$ \\ ${ }^{1}$ Former Department of Nursing, Juntendo University Hospital, Tokyo, Japan; *Corresponding Author: usakonominmi@yahoo.co.jp \\ ${ }^{2}$ Department of Hospital Administration, Juntendo University School of Medicine, Tokyo, Japan \\ ${ }^{3}$ Department of Nursing, Juntendo University Nerima Hospital, Tokyo, Japan
}

Received 6 December 2010; revised 10 February 2011; accepted 15 February 2011.

\begin{abstract}
Since standardization is an important safety measure in healthcare systems, it is essential to systematically assess the effects of introducing new and increasingly complex medical equipment, such as infusion devices. In this study, we compared the effects of standardized polyvinyl chloride (PVC)-free closed-system integrated infusion devices with conventional infusion devices. Specifically, we assessed the safety, work efficiency, user-friendliness, and cost effectiveness of these devices. Compared with conventional infusion devices, integrated PVC-free infusion devices were more expensive to purchase and dispose, but were safer and more user-friendly and efficient. Although it would be preferable to use standardized infusion devices in all hospital departments, their cost may limit their application to departments that use high-risk treatments, where they would be most beneficial.
\end{abstract}

Keywords: Standardized Infusion Devices; Efficiency Assessment; Healthcare Management; Nursing Administration

\section{INTRODUCTION}

The use of infusion devices is complex and diverse, due to not only differences in the pharmacological agents that are infused but also the material of infusion devices and their use with electronic medical equipment, which can lead to medical accidents. According to the Japan Council for Quality Health Care, 92 out of 1440 reports that were received from 204 medical institutions during 2008 involved "management-related items" and "the use of drains and tubes," including extravasations of intravenous infusions [1]. In addition, some previous studies also have reported cases in which the combina- tion of tubes and drugs caused dissolution and elution of the highly toxic substance diethylhexyl phthalate (DEHP) [2-4] and lipid-soluble drugs that caused cracks at the connections of tubes, which caused leakage of the drug [5-7]. After receiving reports of these cases, all medical institutions in Japan have begun regulating the use of infusion devices according to the composition of the devices, such as polyvinyl chloride (PVC)-free, DEHPfree, or conventional materials. However, this regulatory process requires the medical staff to have extensive knowledge about the selection and handling of infusion devices, which depends on many factors, such as the intended purpose of infusion, drug, drip rate, and the patient's condition.

In a previous study, we analyzed the risks that are associated with different pharmacological agents, infusion routes, catheter-related infections, and electronic equipment. Specifically, we selected PVC-free closed-system integrated infusion devices (hereafter referred to as standardized infusion devices) as the reference standard in our hospital and introduced these devices into the chemotherapy department, where strict control of the safety of injections is required. Then, to determine the effectiveness of these devices, we compared the time and cost that was required to prepare these devices with those of conventional devices, which consisted of a combination of an infusion set, a T-shaped stopcock, and an extension tube. The results showed that standardized infusion devices improved work efficiency. However, these devices were not as cost-effective as conventional devices due to the cost of materials and the weight of wastes [8].

In this study, we conducted a more comprehensive study to assess the effectiveness of standardized infusion devices, including their safety and user-friendliness.

\section{SUBJECTS AND METHODS}

This study was conducted in accordance with the guidelines of our institutional review board. 


\subsection{Comparison of the Incidence of Adverse Events before and after the In- troduction of Standardized Infusion Devices}

The safety of standardized infusion devices was assessed by comparing the incidence of adverse events in the medicine department and in the chemotherapy department. The study was conducted for $10 \mathrm{~d}$ before introducing the standardized infusion devices and for $10 \mathrm{~d}$ 2 months afterwards. A check sheet was used to record the number of patients who received an infusion every day and the number of adverse events that occurred. Then adverse events were checked: 1) "Leakage of drug solution due to loosening (unfastening) of the connector of the infusion device”, 2) "Leakage of drug solution due to loosening of the joint between the intravenous needle and the infusion device", 3) "Attempts to disconnect the joint manually were unsuccessful", 4) "There was a crack in the three-way stopcock", 5) "The T-shaped stopcock and the protective cap became dirty", 6) "The connector was no longer sterile after an infusion route was established", 7) "No infusion set was available for the infusion pump that was used", 8) "No infusion set was available for the type of pharmacological agent that was selected". The incidence of adverse events was calculated as the number of adverse events that occurred per patient per day, and the number of adverse events was calculated as the number of days in the survey period divided by the number of patients. The incidence of adverse events before and after the introduction of standardized infusion devices was compared by using Fisher's exact test. The level of significance was defined as 0.05 .

\subsection{Survey of User-Friendliness and Safety}

Between February 21, 2008, and March 5, 2008, an anonymous survey was conducted among 45 nurses who worked in the medicine department and chemotherapy department, where standardized infusion devices had been used for more than 3 months. The survey asked multiple-choice and yes/no questions about the userfriendliness and usefulness of standardized infusion devices, such as the ease of preparing the administration route and precautions that were needed to ensure sterile operation as well as free-response questions about the perceived advantages and desired improvements in standardized infusion devices. Specifically, the userfriendliness of standardized infusion devices was compared with conventional infusion devices by 5 categories: "better", "slightly better", "same”, "slightly worse" and "worse". In addition, the usefulness of the all-in-one nature, PVC-free components, and lock-type system of standardized infusion devices and whether continued use was desired was determined by "yes", "no", or "neither yes nor no" responses.

For ethical reasons, we conducted this survey anonymously by allowing respondents to drop completed questionnaires into a collection bag during a 2 week period. Statistical analysis of the survey responses was performed by using Mann-Whitney $U$ tests with JMP 6 data analysis software. The level of significance was defined as 0.05 .

\section{RESULTS}

\subsection{Comparison of the Incidence of Adverse Events before and after the In- troduction of Standardized Infusion Devices}

The incidence of adverse events before and after the introduction of standardized infusion devices is summarized in Table 1. One hundred forty-seven patients received infusions during the survey period before standardization, while 389 patients received infusions after standardization. The incidence of 4 different adverse events decreased after the introduction of standardized infusion devices. First, the incidence of the adverse event "Leakage of drug solution due to loosening of the joint between the intravenous needle and the infusion device" decreased from 0.00204 events per patient per day before the introduction to 0 afterwards. Second, the incidence of the adverse event "Attempts to disconnect the joint manually were unsuccessful" decreased from 0.00272 events per patient per day before the introduction to 0.00026 events per patient per day afterwards. Third, the incidence of the adverse event "The T-shaped stopcock and the protective cap became dirty" decreased from 0.00272 events per patient per day before the introduction to 0 afterwards. Fourth, the incidence of the adverse event "Leakage of drug solution due to loosening (unfastening) of the connector of the infusion device" decreased from 0.00068 events per patient per day before the introduction to 0.00026 events per patient per day afterwards. No other adverse events occurred before or after the introduction of standardized infusion devices.

\subsection{Survey of User-Friendliness and Safety}

Forty-one out of 45 surveys were returned. Eleven out of the 13 questions about the convenience of infusion devices showed a statistically significant difference between the standardized and conventional infusion devices, which indicated that the standardized infusion devices were very user-friendly (Figure 1). In addition, 
Table 1. Comparison of the incidences of adverse events before and after the introduction of standardized infusion devices.

\begin{tabular}{|c|c|c|c|c|c|}
\hline Adverse events & Before & After & Before & After & \\
\hline Number of patients who received infusions during the 10 -day survey period & 147 & 389 & $\begin{array}{r}\text { Number o } \\
\text { pati }\end{array}$ & $\begin{array}{l}\text { currences/ } \\
\text { day }\end{array}$ & \\
\hline $\begin{array}{l}\text { 1) Leakage of drug solution due to loosening (unfastening) of the connector of the infu- } \\
\text { sion device. }\end{array}$ & 1 & 1 & 0.00068 & 0.00026 & \\
\hline $\begin{array}{l}\text { 2) Leakage of drug solution due to loosening of the joint between the intravenous needle } \\
\text { and the infusion device. }\end{array}$ & 3 & 0 & 0.00204 & 0.00000 & $*$ \\
\hline 3) Attempts to disconnect the joint manually were unsuccessful (strong union). & 4 & 1 & 0.00272 & 0.00026 & $*$ \\
\hline 4) There was a crack in the three-way stopcock. & 0 & 0 & 0.00000 & 0.00000 & \\
\hline 5) The T-shaped stopcock and the protective cap became dirty. & 4 & 0 & 0.00272 & 0.00000 & $* *$ \\
\hline 6) The connector was no longer sterile after an infusion route was established. & 0 & 0 & 0.00000 & 0.00000 & \\
\hline 7) No infusion set was available for the infusion pump that was used. & 0 & 0 & 0.00000 & 0.00000 & \\
\hline 8) No infusion set was available for the type of pharmacological agent that was selected. & 0 & 0 & 0.00000 & 0.00000 & \\
\hline
\end{tabular}

Before: Before the introduction of the use of standardized infusion devices; After: After the introduction of the use of standardized infusion devices; * $\mathrm{p}<$ $0.01 * *=\mathrm{p}<0.05$.

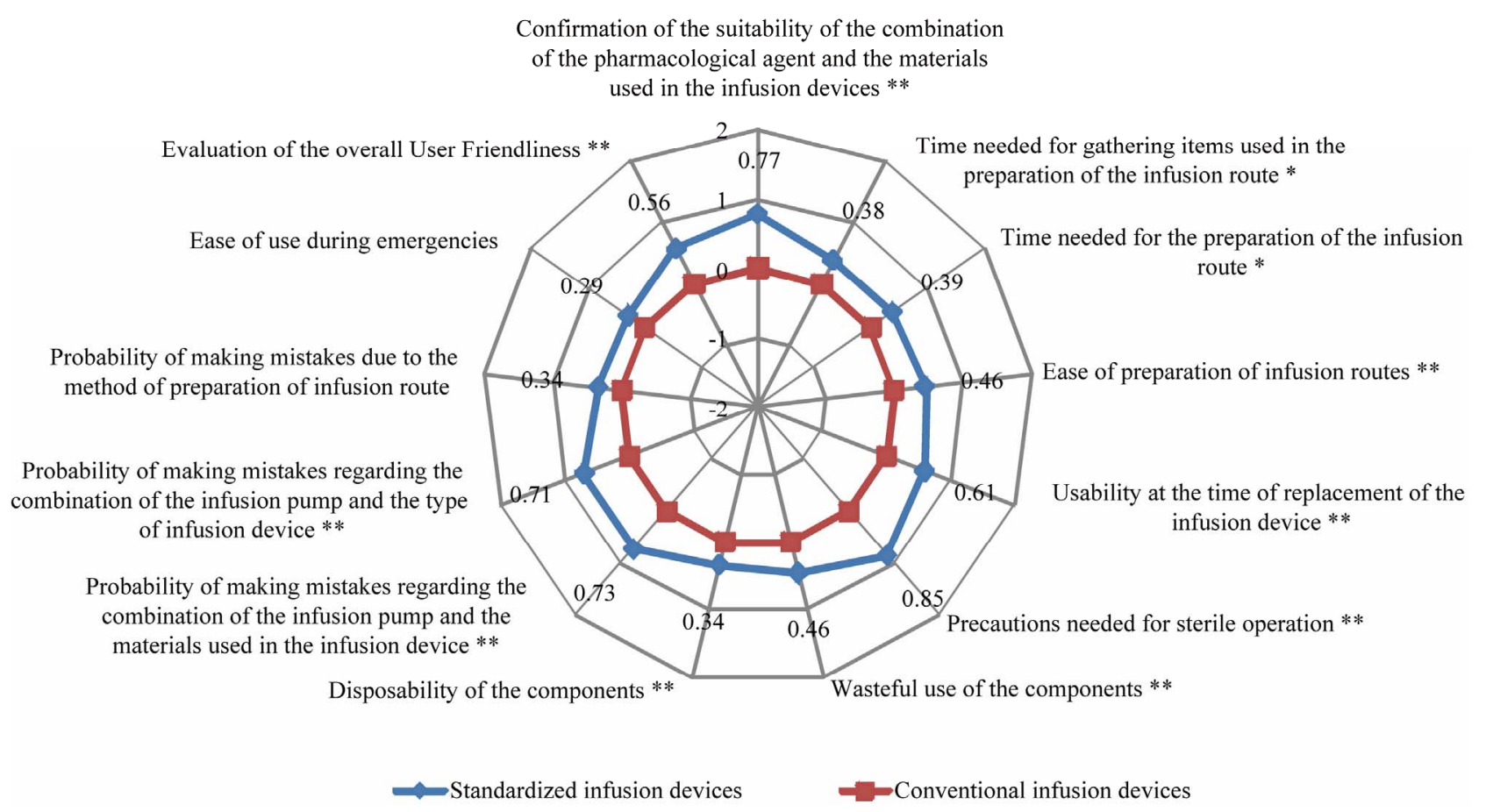

Figure 1. Comparison of the user-friendliness of conventional and standardized infusion devices.

$63.4 \%$ of respondents found the all-in-one nature of standardized infusion devices to be useful, 95.1\% stated that their PVC-free properties were useful, $80.5 \%$ found the lock-type system of the devices to be useful, and $70.7 \%$ indicated that they desired to continue using the standardized infusion devices (Figure 2).

The following responses were listed as perceived advantages of standardized infusion devices: ease of preparing the administration route; robust connection; no need to select administration routes for every drug; ease of tying the side tube without leakage of the drug solution; no need to select a compatible pump for the infusion device; no risk of selecting the incorrect pump setting; and no need for a protective cap for the T-shaped stopcock. In addition, in the chemotherapy department, the following benefits were repeatedly emphasized: no leakage of the drug solution at the connection between the intravenous needle and the infusion device; no risk 


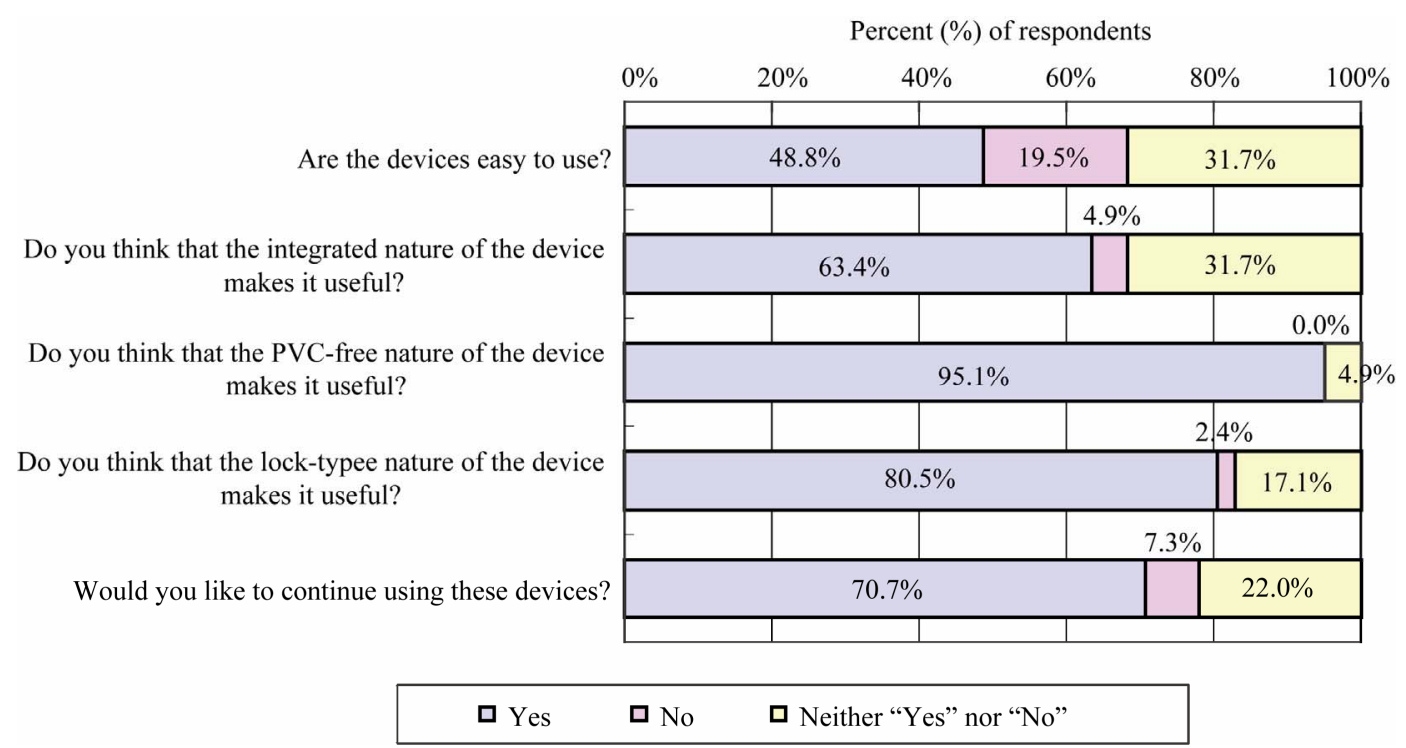

Figure 2. Comparison of the usefulness of conventional and standardized infusion devices.

of selecting an inappropriate infusion device for each drug; the ability to manually adjust the drip rate even when infusion sets for pumps are used; and less time needed to establish an intravenous route.

The following improvements were desired in standardized infusion devices: simpler evacuation of air when connecting the side tube, miniaturization of the structure that connects the intravenous needle to the infusion device, improved description of the device on the package, and a way to adjust the length of the infusion device.

\section{DISCUSSION}

\subsection{Safety Assessment}

Our results showed that the incidence of adverse events involving leakage of drug solutions decreased from an incidence of 0.00204 per patient per day before the introduction, to 0 after the introduction of standardized infusion devices. Although the possibility of occurrence of an incidence of drug solution leakage is very small, the preparation of infusion routes is a routine method in our hospital. Therefore, we consider this result to be significant. Furthermore, this result is consistent with our findings in a previous study [8]. Leakage of a drug solution from an infusion device is a significant problem in intravenous pharmacotherapy because the amount of drug that enters the body will be less than desired and cutaneous disorders may develop as a result of exposure to the drug solution. Moreover, it is pointed out that the additional medical costs are caused by incidents [9]. Previous studies have demonstrated that closed-system devices, such as the standardized infusion devices in this study, reduce the risk of infection [10]. Our results also showed that the introduction of standardized infusion devices reduced the incidence of adverse events involving manual operation and loss of sterility, which suggested that these devices decrease the risk of infection during their preparation and at the time of connection. Collectively, these results demonstrated that standardized infusion devices are safer than conventional infusion devices. Furthermore, we think that an extensive comparison of the incidence of adverse events by increasing the number of samples is required in the future.

\subsection{Assessment of User-Friendliness}

The results of the survey showed that standardized infusion devices were more user-friendly than conventional infusion devices in all aspects of their use, including time, operation, cleaning, and risk of adverse events. Moreover, the favorable responses to questions about precautions that are needed for cleanliness of operation and the ease of operation at the time of replacement combined with the fact that standardized infusion devices can be operated with precision suggested that anyone can use these devices, even in emergencies. The simple operation of standardized infusion devices is important because the most common causes of accidents involving pharmacological agents during 2008 were "lack of verification," "insufficient knowledge," and "mistaken judgment," while the most common causes of tube-related accidents were "lack of verification" and "mistaken judgment" [1]. To prevent adverse reactions between the pharmacological agent and the materials used in the infusion device and pump, it is necessary to know how to select materials and instruments that are appropriate for particular drugs. As a result, continuing 
education about drugs and the properties of medical materials is necessary. However, since standardized infusion devices are made with PVC-free components, it is not necessary to use a specific infusion device for specific drugs. As a result, standardization not only simplifies the practice but also the educational requirements of infusion management.

These conclusions are supported by the finding that most of the respondents in this study considered the physical characteristics of standardized infusion devices useful and desired to continue using them. Moreover, their free responses indicated that standardized infusion devices are safe, effective, and easy to use.

\subsection{Assessment of Usability}

In addition to safety and user-friendliness, we also assessed the usefulness of standardized infusion devices in terms of their work efficiency and cost-effectiveness. In our previous study, we measured the work efficiency of infusion devices by measuring the time that was needed to prepare an infusion device. We found that the preparation time for standardized infusion devices (55.8 (12.0) s) (mean (SD) was shorter than that for conventional infusion devices (96.1 (22.4) s) (Figure 3). In the chemotherapy department, the preparation time of conventional and standardized infusion devices was (97.0 (22.0) $\min \cdot \mathrm{d}^{-1}$ ) and (22.0 (3.0) $\left.\mathrm{min} \cdot \mathrm{d}^{-1}\right)$, respectively. Thus, the introduction of standardized infusion devices improved the work efficiency of infusions by decreasing the time that was required to select different products for each drug [8].

In our previous study, we also determined the cost-effectiveness of infusion devices by performing a cost simulation of the purchase price of the devices, labor costs for improving their work efficiency, and the amount of medical waste they generated. We showed that the cost of conventional infusion devices was $¥ 280,405$ (Japanese Yen) for central venous routes and
$¥ 1,003,019$ for peripheral venous routes. In addition, we predicted that the purchase price of standardized infusion devices is higher than that for conventional devices, despite the costs of medical waste disposal and labor associated with improving work efficiency (Table 2) [8].

As a result, although standardized infusion devices are more expensive than conventional infusion devices, the standardized devices are safer and more user-friendly and efficient. Ideally, standardized infusion devices should be used in all hospital departments; however, their higher cost may limit its introduction to departments that use high-risk treatments, such as the chemotherapy department, intensive care unit, and pediatric ward, where they would be most beneficial.

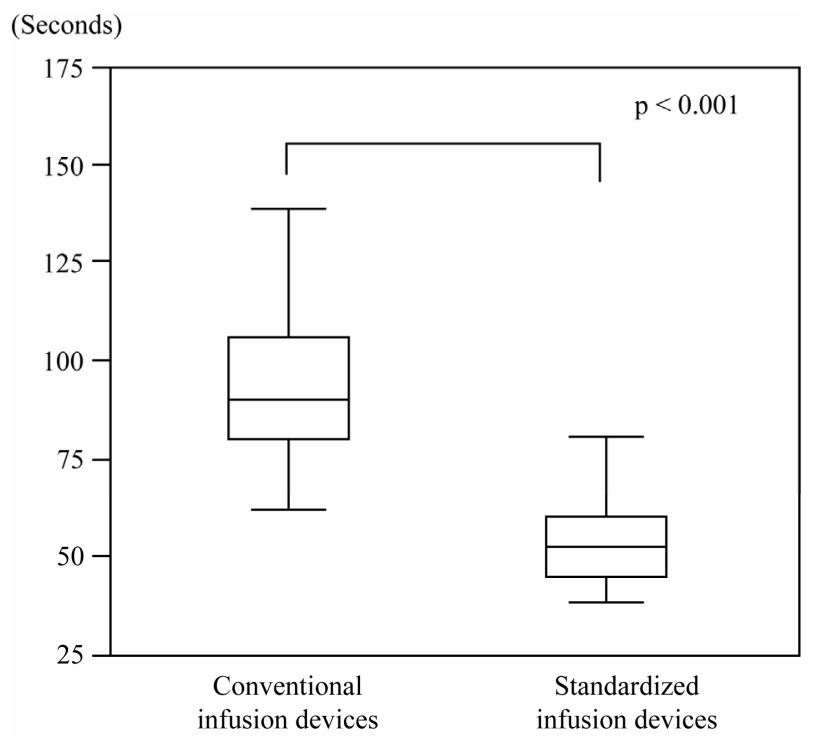

Figure 3. Comparison of the time required to prepare a fluid infusion route using conventional and standardized infusion devices. (Sugita, S., et al. Evaluation of the cost effectiveness and work efficiency of the use of standardized infusion devices. Japanese Journal of Health Care Management, 9(4), 2009).

Table 2. Cost simulation.

\begin{tabular}{|c|c|c|c|c|c|c|c|c|c|c|}
\hline & & $\begin{array}{c}\text { Price } \\
\text { (Japanese } \\
\text { Yen/device) }\end{array}$ & $\begin{array}{c}\text { Disposal } \\
\text { costs } \\
\text { (Japanese } \\
\text { Yen/100 } \\
\text { devices) }\end{array}$ & $\begin{array}{c}\text { Number } \\
\text { (devices/ } \\
\text { month) }\end{array}$ & $\begin{array}{c}\text { Price } \\
\text { (Japanese } \\
\text { Yen/month) }\end{array}$ & $\begin{array}{c}\text { Disposal } \\
\text { costs } \\
\text { (Japanese } \\
\text { Yen/month) }\end{array}$ & $\begin{array}{l}\text { Total costs } \\
\text { (Japanese } \\
\text { Yen/month) }\end{array}$ & $\begin{array}{l}\text { Standardized- } \\
\text { Conventional } \\
\text { (Japanese } \\
\text { Yen/month) }\end{array}$ & $\begin{array}{l}\text { Eliminated } \\
\text { personnel } \\
\text { costs } \\
\text { (Japanese } \\
\text { Yen/month) }\end{array}$ & $\begin{array}{l}\text { Predicted costs after } \\
\text { the introduction of } \\
\text { the use of the } \\
\text { standardized type of } \\
\text { devices (Japanese } \\
\text { Yen/month) }\end{array}$ \\
\hline $\begin{array}{l}\text { Central } \\
\text { venous }\end{array}$ & Conventional & 1,510 & 597 & 750 & $1,132,500$ & 4,475 & $1,136,975$ & & & \\
\hline infusion & Standardized & 1,900 & 663 & 750 & $1,425,000$ & 4,974 & $1,429,974$ & +292,999 & $-12,594$ & $+280,405$ \\
\hline $\begin{array}{l}\text { Peripheral } \\
\text { venous }\end{array}$ & Conventional & 480 & 454 & 9000 & $4,320,000$ & 40,856 & $4,360,856$ & & & \\
\hline infusion & Standardized & 600 & 438 & 9000 & $5,400,000$ & 39,438 & $5,439,438$ & $+1,078,582$ & $-75,563$ & $+1003,019$ \\
\hline
\end{tabular}

Sugita, S., et al. Evaluation of the cost effectiveness and work efficiency of the use of standardized infusion devices. Japanese Journal of Health Care Management, 9(4), 2009. 
Due to the increasing complexity of drug regimens and drug-delivery devices, it is increasingly difficult to perform cost-benefit analyses of all new medical equipment. However, comprehensive assessments are important to effectively implement and manage health safety measures. In this study, we analyzed 4 different aspects of infusion devices, which are considered to be comprehensive. Moreover, we consider that our future tasks are to introduce this study results to another hospital and then to be calculated based on the situation in that hospital.

To enhance the safety of healthcare services, medical devices should be standardized. In addition, standardization should be applied at all medical institutions nationwide. Furthermore, pharmaceutical and medical equipment companies should work with medical institutions to improve the safety of drug delivery systems to help develop safer and more effective medical care.

\section{CONCLUDING REMARKS}

To determine the overall effectiveness of using standardized infusion devices, we assessed their safety, work efficiency, user-friendliness, and cost-effectiveness. Our results showed that standardized infusion devices are more expensive than conventional infusion devices; however, standardized infusion devices are safer and more user-friendly and efficient. Ideally, standardized infusion devices should be used in all hospital departments, but their higher costs may limit their use to departments that use high-risk treatments, where they would be most beneficial. This study highlights the advantages of standardizing medical equipment, which is important for improving healthcare management and administration.

\section{REFERENCES}

[1] The Japan Council for Quality Health Care (2009) The Project to Collect Medical Near-Miss/Adverse Event In- formation. 16th Report, 24 March 2009, Division of Adverse Event Prevention.

[2] Ministry of Health, Labour and Welfare of Japan (2002) Pharmaceuticals and medical devices safety information No. 182.

http://www.mhlw.go.jp/houdou/2002/10/h1031-1a.html\# $\underline{2}$

[3] Health Canada (2001) DEHP in medical devices: An exposure and toxicity assessment.

http://www.hc-sc.gc.ca/dhp-mps/md-im/activit/sci-consul t/dehp/sapdehp rep gcsdehp rap 2001-04-26-eng.php

[4] U.S. Food and Drug Administration (2001) Safety Assessment of $\mathrm{Di}(2$-ethylhexyl)phthalate (DEHP) released from PVC medical devices. http://www.tracingpapers.org/article.php3?id article $=38$

[5] Ministry of Health, Labour and Welfare of Japan (2003) Pharmaceuticals and medical devices safety information No.196.

http://www.mhlw.go.jp/houdou/2003/12/h1225-4.html\#g ai2

[6] Nakao, M., Yamanaka, S., Iwata, M., Nakashima, M. and Onji, I. (2003) The cracks of polycarbonate three-way stopcocks are enhanced by the lubricating action of fat emulsion of propofol. Japanese journal of anesthesiology, 52, 1243-1247.

[7] Nakao, M., Yamanaka, S., Harada, A. and Onji, I. (2000) Cracks of polycarbonate three-way stopcock are caused by fat emulsion not by propofol. Japanese journal of anesthesiology, 49, 802-805.

[8] Sugita, S., Okada, A., Hori, S., Aida, H., Tamura, T., Suzuki, H., et al. (2009) Evaluation of the cost effectiveness and work efficiency of the use of standardized infusion devices. Japanese Journal of Health Care Management, 9, 519-523.

[9] Kobayashi, M., Ikeda, S. and Muto, M. (2006) The Estimation of medical cost incurred by incidents and adverse events. Japanese Journal of Health Care and Social Welfare, 16, 85-96.

[10] Yorioka, K., Oomaki, M., Sawa, H., Oie, S. and Kamiya, A. (2005) Bacterial contamination of three-way stopcock and closed-type infusion device used in central venous catheter. Japanese Journal of Environmental Infections, 20, 272-274. 